\title{
Simulation Research on Neutral Grounding Modes of Marine High Voltage Power System Wang Haoliang ${ }^{1,}$,Z,Zhang Chunlai, ${ }^{1,}$, Li Meng, Lu Kun \\ ${ }^{1}$ Marine Engineering College, Dalian Maritime University, Dalian, 116026, China \\ aemail: hl.wang0910@hotmail.com, bemail:zclzyc@163.com
}

Key words: Marine high voltage power system ; Grounding mode ; Grounding current ; Simulation

\begin{abstract}
Based on the characteristics of marine power system, the paper takes marine high voltage power system as the research object to research the five kinds of neutral grounding modes. The simulation model is built by MATLAB/SIMULINK to analyze the difference of the different neutral grounding modes. By comparing the results of simulation data, it shows that the high resistance neutral grounding mode is fit for the marine high voltage power system.
\end{abstract}

\section{Introduction}

With the advantages of large volume and low freight as well as the strong adaptability to the cargo, shipping becomes the main mode of transportation in the international trade. In this background, the ship, as the main means of transport, should inevitably develop toward the direction of the large-scale, quick running and automation. Because of the power bottleneck of the low voltage generator, economic problems and the restriction on the installation of space, low voltage power system has been unable to meet the requirements of the production. So the high voltage power system is widely used on the large vessel.

Marine power system is a vital component of the ship, which has very strict requirements in safe operation. Traditional marine low voltage power system generally adopts the method of isolated neutral, this method has advantage that can support the continuous power supply. However, with the increasing of the power voltage, grounding current will be increased and also the probability of arc grounding overvoltage will be increased, which makes it easier for the power network caused by the overvoltage and overcurrent to become paralysis. It is a serious threat to the safety of the marine power system. So, the method of isolated neutral is no longer applicable to marine high voltage power system .This makes the research of neutral grounding system for marine high voltage power system become an important technical problem.

\section{The mode of neutral grounding}

Neutral point of power system refers to the neutral point of transformer or Y-connected generator. The mode of neutral point grounding is a comprehensive and complex problem which involves the system insulation level, communication interference, grounding protection method, the setting of protection, voltage grade and the structure of power system, etc.

The grounding mode of the marine high voltage power system can be divided into neutral directly grounding, neutral point via arc suppression coil grounding, high resistance neutral grounding, and low resistance neutral grounding, etc. By comparing various grounding modes and considering comprehensive factors, this paper puts forward a mode of neutral grounding which is suitable for marine high voltage power system.

\section{Isolated neutral}

The most prominent advantage of isolated neutral mode is that the system can still continue to run normally when single-phase is grounded. When a restorable single-phase grounding fault occurs, the system can recover automatically. When single-phase grounding fault cannot recover, the crew can generally solve the fault circuit within several hours. The isolated neutral mode has high level of power supply reliability. Moreover, this operation is low cost and has a simple technology. So it 
is widely used on the marine low voltage power system.

\section{Neutral directly grounding}

Neutral directly grounding is a mode that neutral point is directly connected to the hull. In this kind of power system, if the single-phase grounding fault occurs, the neutral-point and single-phase ground will constitute a loop and form a short circuit, the single phase short-circuit will make the fuse on installation fusing or automatic switch trip. Then it will remove the fault point. Comparing with the isolated neutral mode, this operation effectively prevents an intermittent arc grounding overvoltage and decreases the possibility of arc grounding overvoltage when the single-phase grounding fault appears. Beside, the voltage of neutral-point will remain zero when single-phase grounding fault occurs. So there will not be the issue of discharging the earth capacity.

\section{Neutral point via arc suppression coil grounding}

The mode of neutral point via arc suppression coil grounding is refers to the mode that an arc suppression coil is in series between neutral-point and hull. The arc suppression coil is an inductor that separates the direct connection between the neutral-point and the ground. Although the isolated neutral power system could run when the single phase grounding fault occurred, the operation of isolated neutral is prone to produce overvoltage of arc light grounding or overvoltage of arc grounding. This mode can solve the problem when the single-phase grounding fault occurs, the voltage of neutral point to ground from zero to phase voltage, namely: $U_{\mathrm{N}}=-U_{\mathrm{A}}$. Arc suppression coil will produce inductive current $I_{\mathrm{L}}$. The formula is as follows:

$$
\begin{aligned}
& I_{\mathrm{L}}=\frac{-U_{\mathrm{A}}}{j \omega L} \\
& L: \text { value of the arc suppression coil inductance. }
\end{aligned}
$$

\section{High resistance neutral grounding}

The mode of high resistance neutral grounding refers to put a higher value resistance between the hull and the neutral point .When single-phase grounding fault occurs, the resistance is an energy dissipation device and will release a large amount of electric charged in the capacitor, so it can be effectively prevent overvoltage of arc light grounding and resonance overvoltage.

Based on the definition of high resistance neutral grounding system:

$$
I_{\mathrm{RN}}>I_{\mathrm{C}} \text {, namely: } R_{\mathrm{N}} \leq \frac{10^{6}}{3 w C_{0}}
$$

$I_{\mathrm{RN}}$ : all current of high resistance grounding through the power system , A;

$I_{\mathrm{C}}$ : system relatively capacitive current, $\mathrm{A}$;

$R_{\mathrm{N}}$ : the value of grounding resistance, $\Omega$;

$C_{0}$ : The capacitance of power system on each phase, $\mu \mathrm{F}$.

If the single grounding fault appears in this system, the grounding fault current should be the sum of current through the high resistance and the relatively capacitive current, the formula is:

$$
I_{\mathrm{jd}}=\sqrt{I_{\mathrm{RN}}^{2}+I_{\mathrm{C}}^{2}}=\sqrt{\left(\frac{U}{\sqrt{3} R_{\mathrm{N}}} \times 10^{3}\right)^{2}+\left(\sqrt{3} U \omega C_{0} \times 10^{-3}\right)^{2}}
$$

$I_{\mathrm{jd}}$ : fault current of Single- phase ground , A;

$U$ : rated line voltage of power system, $\mathrm{kV}$;

\section{Low resistance neutral grounding}

Low resistance neutral grounding usually adopts the resistance value of $1 \sim 15 \Omega$, the values need to be able to guarantee the largest single phase grounding fault current is less than the largest interphase fault current. This grounding method can be below the safe limit over-voltage of arc light grounded. 


\section{The simulation research}

As shown in the figure 1, the marine high voltage power system simulation model of a 10,000 TEU containership is built. The model uses a simplified three-phase synchronous generator as the power source of the power system. And the induced voltage of the generator is controlled by the excitation regulator, mechanical torque is supplied by the diesel engine.

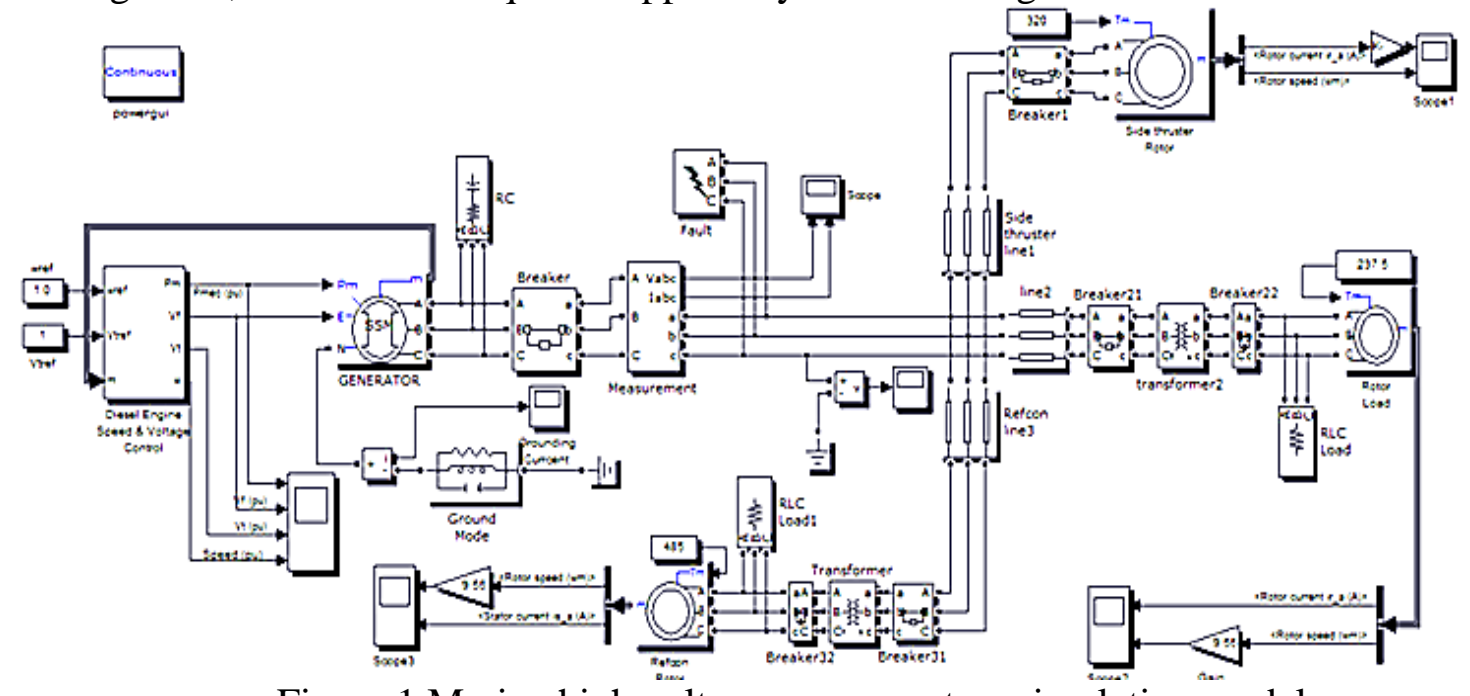

Figure 1 Marine high voltage power system simulation model

\section{Comparison of the neutral point grounding ways}

\section{The mode of Isolated neutral}

The ground fault of phase A occurs when $T=0.5 \mathrm{~s}$, it can be seen from the figure 2(a), the grounding current is only about $0.3 \mathrm{~A}$. The figure 2(b) shows that the voltage of non-fault phase to the ground suddenly rise to $\sqrt{3}$ times after the single phase grounding, so the insulation level of the demand is higher.

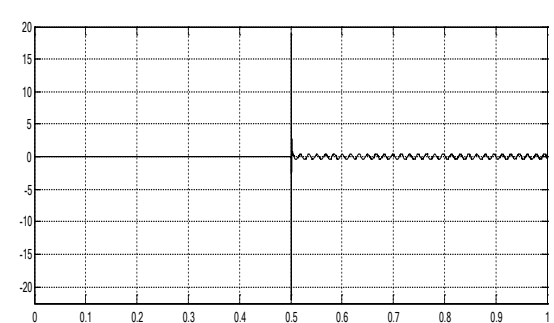

Figure 2(a) The grounding current

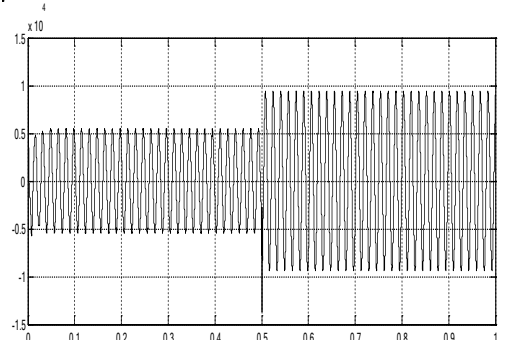

Figure 2(b) The voltage of non-fault phase

\section{The mode of Neutral directly grounding}

Under the condition of the neutral directly grounding, single-phase earth fault occurs when $T=$ $0.5 \mathrm{~s}$, it can be seen from figure3(a) that the shock value of grounding current reaches more than $4000 \mathrm{~A}$, and the peak value reaches more than $2000 \mathrm{~A}$ when the system is stable. Which inevitably lead to the action of the relay protection device to cut off the fault circuit.

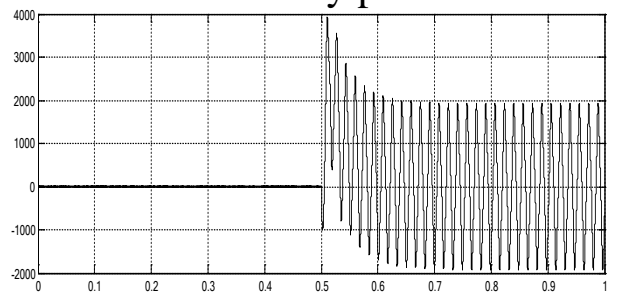

Figure 3(a)The grounding current

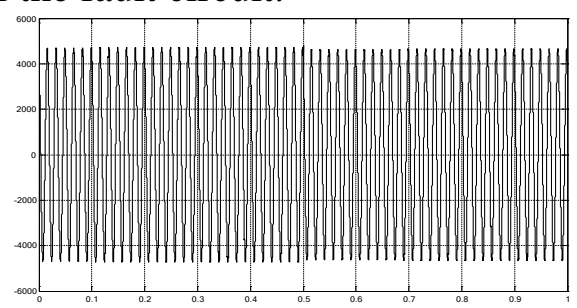

Figure 3(b)The voltage of non-fault phase 


\section{The mode of neutral point via arc suppression coil grounding}

As shown in figure 4(a), the effective value of fault current is very small, about $0.5 \mathrm{~A}$. The system will not produce the problem such as over-voltage and over-current.

When the single-phase grounding fault occurs, the system fault current is equal to the vector sum of inductive current and the capacitive current:

$$
I_{\mathrm{D}}=I_{\mathrm{L}}+I_{\mathrm{C}}
$$

Calculation formula for inductive current is: $I_{\mathrm{L}}=\frac{-U_{\mathrm{A}}}{j \omega L}$

Calculation formula for capacitive current is: $I_{\mathrm{C}}=\omega C U_{\varnothing}$

The system's grounding capacitive value is: $C_{\sum_{0}}=2.0 e^{-7} \mathrm{~F}$

So it can be calculated that the value of whole compensation inductance: $L=11.727 \mathrm{H}$.

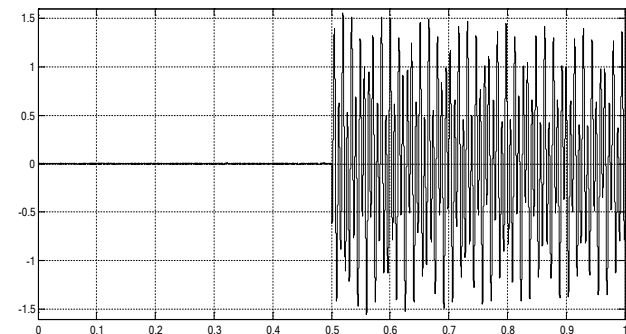

Figure 3(a)The grounding current

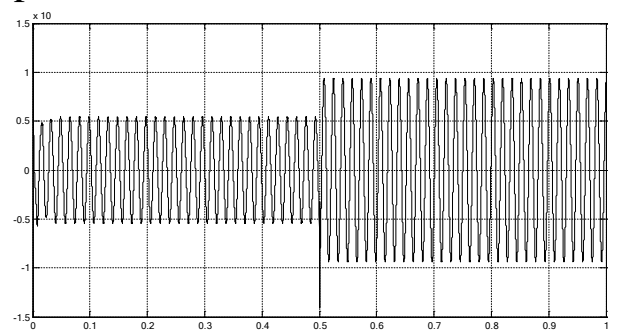

Figure 3(b)The voltage of non-fault phase

The mode of high resistance neutral grounding

As shown in figure 4(a), the effective grounding current is about 4A when single-phase ground fault occurs when $T=0.5 \mathrm{~s}$. Because of high resistance value, the grounding current is limited in a controllable range. As shown in figure 4(b), the fault phase to ground voltage is raised to the line voltage.

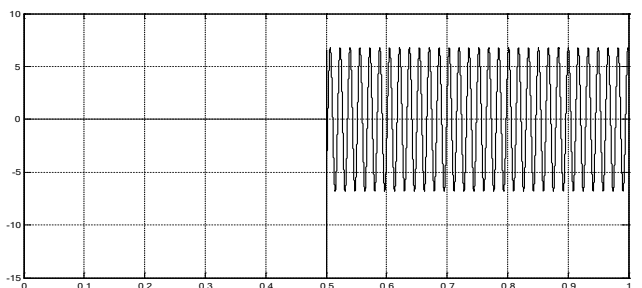

Figure 4(a)The grounding current

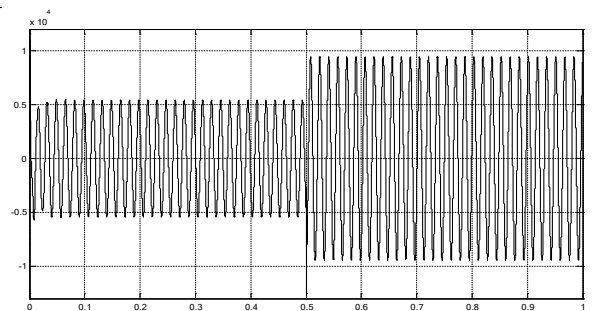

Figure 4(b)The voltage of non-fault phase

The mode of low resistance neutral grounding

As shown in figure 5(a), when the single-phase earth fault occurs, because the resistance connected in series with the fault circuit is small, the grounding current is relatively large.

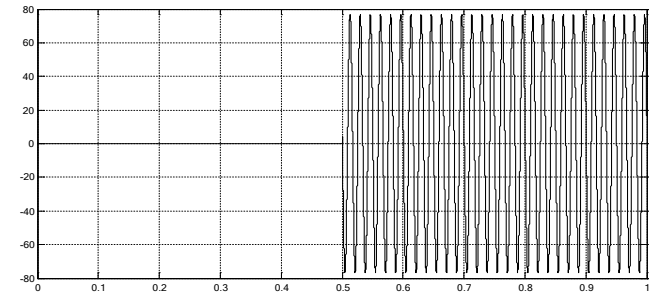

Figure 5(a)The grounding current

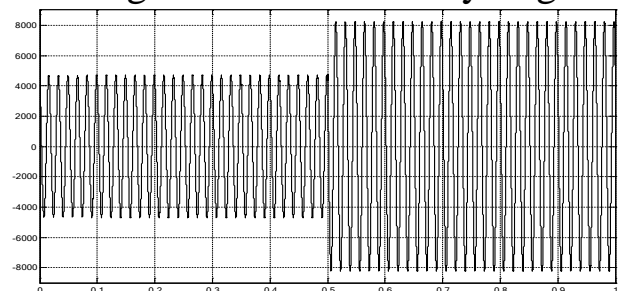

Figure 5(b) The voltage of non-fault phase

\section{Conclusion}

By comparing the five kinds of neutral grounding modes, it is obvious that: Firstly, this mode ensures the continuity of power supply, which improves the stability of marine power system. Secondly, it controls the grounding current within 10A when single-phase ground fault occurs, otherwise, it has a small interference to the nearby communication system, and will not generate overvoltage and overcurrent, the possibility of developing into more serious fault is very small. The 
level of grounding current is easy to for the protection of the relay protection device. Finally, the cost of high resistance grounding is relatively low compared with other modes. So the high resistance neutral grounding mode is the most ideal mode for marine high voltage power system.

\section{References}

[1] Zhu Yanbing. Design and Research of the Integrated Protection of Marine High-voltage Electric Power System [D].Dalian: Dalian Maritime University.2012.

[2] Zhang Lingjie. Simulation Research of "Pacific" Marine High-voltage Electric Power System [D].Dalian: Dalian Maritime University.2013.

[3] Wang Yan. Research on Neutral Grounding Pattern and Over-voltage of Vessel Power System Medium Voltage Network [D].Wuhan: Wuhan University of Technology.2011.

[4] Wang Jie. Research on Neutral Grounding Pattern’s Operating Characteristic and Over-voltage problem of Independent Power System Medium Voltage Network [D](Master's Thesis).Wuhan: Huazhong University of Science\&Technology.2008.

[5] Shott HS, H A. eterson. Criteria for neutral stability of wye-grounded-primary broken delta-secondary transformer circuit [J]. Transaction on AIEE. 1941.24 (4): Yunge Li, Wei Shi; A Systematical Method for Suppressing Ferroresonance at Neutral-Grounded Substations [J]. IEEE Transactions on Power Delivery. 2003.7.18(3):1009 1014.

[6] Milan Graovac, Reza Iravani, Fast Ferroresonance Suppression of Coupling Capacitor Voltage Transformers [J], IEEE Transactions on Power Delivery, 2003 18(1):158 163. 